\title{
Adsorption of monoterpene alcohols at the water-air interface
}

\author{
Andrzej Lewandowski ${ }^{1}$ [ $\cdot$ Katarzyna Szymczyk $^{1}$
}

Received: 30 November 2018 / Revised: 6 January 2019 / Accepted: 8 January 2019 / Published online: 18 January 2019

(c) The Author(s) 2019

\begin{abstract}
The measurements of the surface tension of aqueous solutions of 10 monoterpene alcohols, MAs: (-)- $\beta$-citronellol, geraniol, tetrahydrolinalool, linalool, (-)-menthol, (-)-terpinen-4-ol, p-cymene-8-ol, $\alpha$-terpineol, isoborneol and (-)-borneol were made at $\mathrm{T}=293 \mathrm{~K}$. On the basis of the obtained results the values of the adsorption constant, the excluded area per molecule at the interface and the interaction parameter were determined by fitting the experimental data to various adsorption models. Next the adsorption free energy of studied MAs was determined and discussed in terms of the preferred orientation of studied MAs at the water-air interface and molecular structure of MAs.
\end{abstract}

Keywords Adsorption · Water-air interface $\cdot$ Monoterpene alcohols $\cdot$ Interaction parameter $\cdot$ Adsorption free energy

\section{Introduction}

Fragrances constitute one of the most significant components of cosmetic, personal or home care products formulations. Consumers are attracted by pleasant smell which in turn, is associated with effective action due to perfume molecules release or evaporation from surfaces. Fragrances are largely built of apolar molecules with low solubility in water. Moreover, in the absence of significant amounts of such co-solvents as ethanol, their solubilisation requires surfactants when they are applied in water. In this case at the equilibrium state the solutes are distributed between the aqueous phase and the hydrophobic pseudo-phase formed by micelles, respectively (Saeki 2019; Braga et al. 2018; He et al. 2018; Kaur et al. 2018; Bicchi et al. 2018; Priebe and Daugulis 2018; Fieber et al. 2018).

Fragrance is frequently treated as a single component in formulated consumer products. As a matter of fact, it is the most complex additive to the formulation from the chemical point of view. Its composition can have over 100 compounds such as essential oils, their isolates and some synthetic aroma chemicals (D'Auria et al. 2017; Costa et al. 2015). Thus a number of reactions can proceed between the

Andrzej Lewandowski

andrzej.lewandowski@poczta.umcs.lublin.pl

1 Department of Interfacial Phenomena, Faculty of Chemistry, Maria Curie-Skłodowska University, Maria

Curie-Skłodowska Sq. 3, 20-031 Lublin, Poland fragrance components and/or the base which cause undesirable changes as far as solubility, clarity, colour, viscosity or $\mathrm{pH}$ of products are concerned. These reactions can be accelerated also by the environmental conditions such as temperature, humidity and light which are often simulated in a laboratory so as to study fragrances compatibility and stability for their applications. One should keep in mind that the impact of the fragrance is mostly proportional to its concentration, that is it depends on the selection of type and concentration of perfumery raw materials (PRMs) in order to supply enough quantities which evaporate into the gas phase (headspace) to be detected. Accordingly, the performance of perfumes in consumer products depends not only on the intrinsic olfactory properties of PRMs, such as their odour thresholds, but also on their physicochemical properties, e.g. vapour pressure and solubility in a base formulation (Costa et al. 2015; Sell 2006). For instance, appropriate selection of type and composition of surfactants can be used to tune the equilibrium partitioning of PRMs between aqueous and micellar phases with a direct impact on the equilibrium headspace concentrations of PRMs above aqueous surfactant solutions (Fieber et al. 2018). Furthermore, the overall performance of perfumes in consumer products is determined not only by the thermodynamically controlled equilibrium partitioning of individual PRMs into the gas phase but also by kinetic factors, i.e. the rate of release and evaporation of PRMs from a base formulation or from surfaces they are applied on. Accordingly, the enhanced delivery of perfumes to interfaces is of vital importance for their effectiveness in 
a wide range of home and personal care products (Bradbury et al. 2016; Penfold et al. 2017). Therefore, for full description of PRMs properties in a fragrance blend and their performance in consumer products, it is necessary to explain their adsorption at various interfaces, particularly at the water-air one.

Monoterpene alcohols (MAs) are primary, secondary or tertiary alcohols with a hydrocarbon backbone consisting of two isoprene units. They are isolated from plant essential oils or obtained by (bio)chemical synthesis methods and constitute a very important group of PRMs (Sato et al. 2018; Bonikowski et al. 2016). As the reports about the surface activity of MAs are scarce in the literature, the aim of this study was to investigate the influence of the molecular structure of monoterpene alcohols on their adsorption at the water-air interface. To achieve it, measurements of the surface tension at $\mathrm{T}=293 \mathrm{~K}$ were made for the aqueous solutions of ten MAs, including acyclic, monocyclic, bicyclic and aromatic compounds. The values of the adsorption constant $K$, the excluded area per molecule at the interface $\alpha$, and the interaction parameter $\beta$ were determined by fitting the experimental data to adsorption models to calculate adsorption free energy, $E_{a}$. The effect of the molecular structure of MAs on the values of the parameters of the models was discussed in terms of the preferred orientation of studied MAs at the water-air interface.

\section{Experimental}

(-)- $\beta$-Citronellol $(\geq 99 \%)$, geraniol ( $\geq 98 \%)$, tetrahydrolinalool ( $\geq 99 \%)$, linalool ( $\geq 99.5 \%)$, (-)-menthol ( $\geq 99 \%)$, (-)-terpinen-4-ol ( $\geq 96 \%)$, $\alpha$-terpineol ( $\geq 96 \%)$, isoborneol $(\geq 95 \%)$ and (-)-borneol ( $\geq 98 \%)$ were kindly provided by Pollena-Aroma. p-Cymene-8-ol ( $\geq 95 \%)$ was purchased from
Fig. 1 Molecular structure of studied monoterpene alcohols

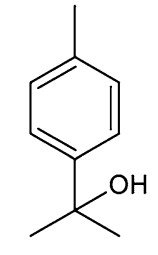

p-Cymene-8-ol

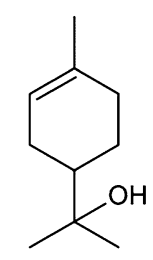

$\alpha$-Terpineol

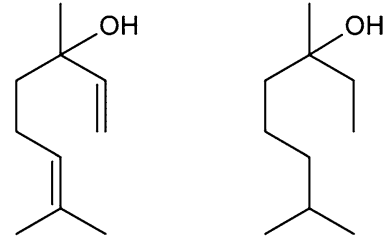

Linalool

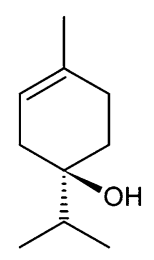

(-)-Terpinen-4-ol

(-)-Menthol
Sigma-Aldrich. The molecular structure of these alcohols is presented in Fig. 1. All fragrance materials were used without further purification and their aqueous solutions were prepared with water deionised by double distillation in a quartz apparatus (Destamat Bi 18E, QCS GmbH). Surface tension measurements were made at the temperature $\mathrm{T}=293 \mathrm{~K}$ by the Wilhelmy plate method with the K100C force tensiometer equipped with a Pt plate (Krüss $\mathrm{GmbH}$ ).

\section{Results and discussion}

Taking into account the measured values of surface tension, $\gamma_{L V}$, of aqueous solutions of monoterpene alcohols (Figs. 2, $3,4,5$ ) firstly the efficiency of adsorption of these alcohols at the water-air interface was considered. A convenient measure of the efficiency of adsorption is the negative logarithm of the concentration of a solute in the bulk phase required to produce a $20 \mathrm{mN} / \mathrm{m}$ reduction in the surface tension of the solvent (water) $\left(p C_{20}\right)$ (Rosen 2004). As results from Figs. 2, 3, 4 and 5 at the studied temperature $p$-cymene-8-ol, isoborneol and borneol do not reduce the surface tension of water to a value of $52.8 \mathrm{mN} / \mathrm{m}$. In the case of other alcohols, as follows from Table 1, the highest efficiency of adsorption is exhibited by (-)- $\beta$-citronellol. As a matter of fact, this is in accordance with the values of free energy change in adsorption at infinite dilution $\left(\Delta G^{o}\right)$ associated with the transfer of alcohols from the solution to the totally covered interface. This energy can be calculated from the relation (Rosen 2004):

$\Delta G^{o}=-\left(p C_{20}+1.74+\frac{20}{2.303 R T \Gamma_{m}}\right) 2.303 R T$

where $R$ is the gas constant, $T$ - the absolute temperature and $\Gamma_{m}$-the maximal value of the Gibbs surface excess concentration $(\Gamma)$.

Tetrahydrolinalool

$(-)-\beta-$ Citronellol<smiles>CC(C)=CCC/C(C)=C/CO</smiles>

Geraniol<smiles>CC1CCC(C(C)C)C(O)C1</smiles><smiles>CC1(C)C2CCC1(C)[C@H](O)C2</smiles><smiles>CC1(C)C2CCC1(C)[C@H](O)C2</smiles>

Isoborneol

(-)-Borneol 


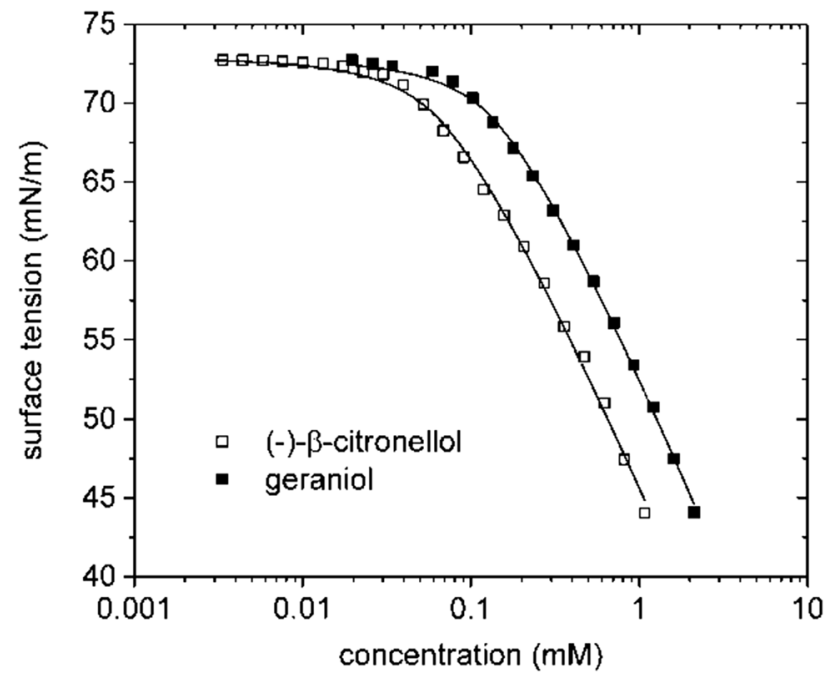

Fig. 2 The values of the surface tension of aqueous solutions of (-)- $\beta$-citronellol and geraniol vs. concentration at $\mathrm{T}=293 \mathrm{~K}$ (points-measured, lines-best fits to the van der Waals adsorption model)

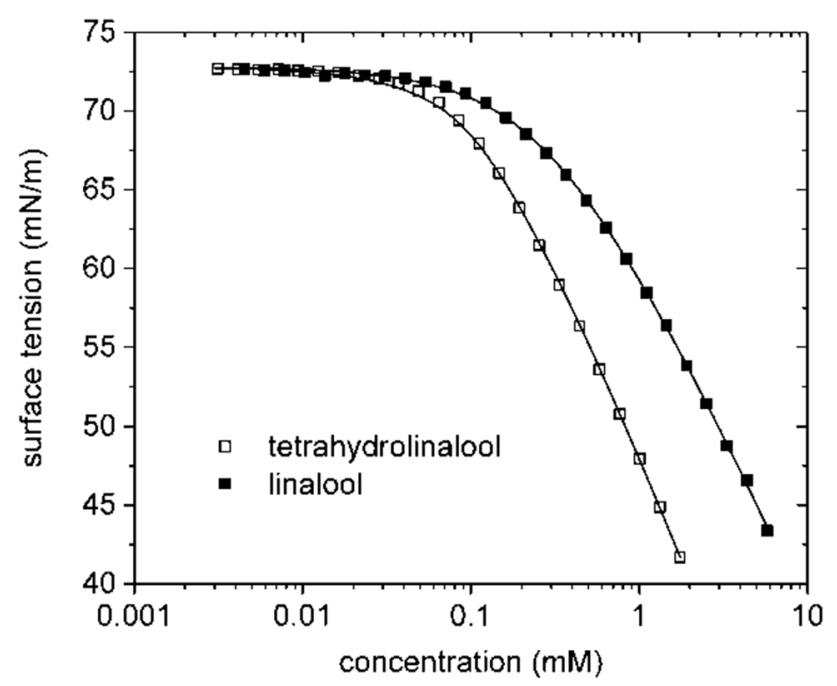

Fig. 3 The values of the surface tension of aqueous solutions of tetrahydrolinalool and linalool vs. concentration at $\mathrm{T}=293 \mathrm{~K}$ (pointsmeasured, lines - best fits to the van der Waals adsorption model)

Assuming that the solute activity coefficient in the studied range of bulk phase concentrations of monoterpene alcohols is close to unity, it is possible to calculate $\Gamma_{m}$ as a limiting slope of the surface tension isotherm, according to the following equation (Rosen 2004):

$\Gamma=-\frac{C}{R T}\left(\frac{d \gamma_{L V}}{d C}\right)_{T}=-\frac{1}{R T}\left(\frac{d \gamma_{L V}}{d \ln C}\right)_{T}=\frac{1}{2.303 R T}\left(\frac{d \gamma_{L V}}{d \log _{10} C}\right)_{T}$

where $C$ is the concentration of MA in the bulk phase. Subsequently, the values of the excluded area per MA molecule at

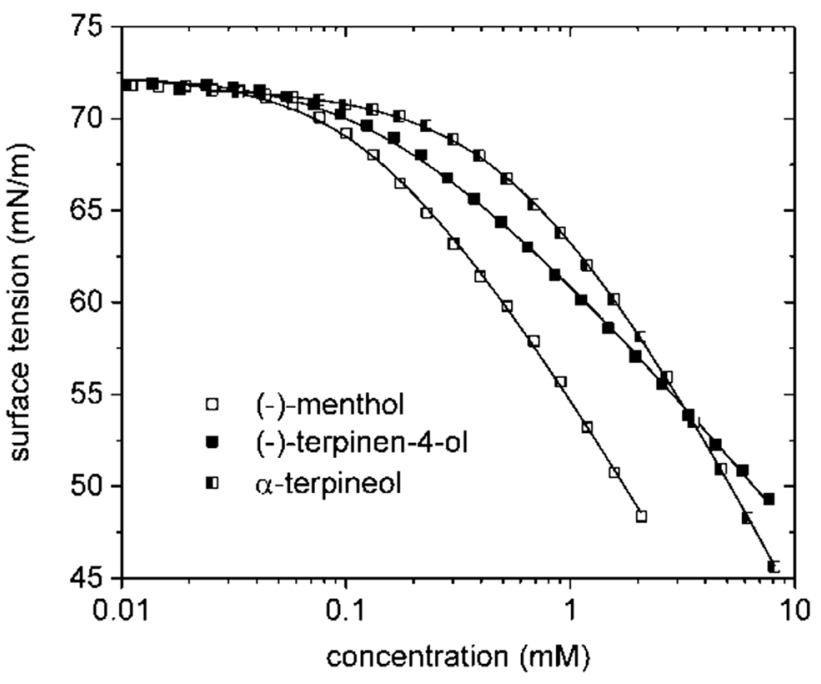

Fig. 4 The values of the surface tension of aqueous solutions of menthol, (-)-terpinen-4-ol and $\alpha$-terpineol vs. concentration at T=293 K (points-measured, lines-best fits to the van der Waals adsorption model)

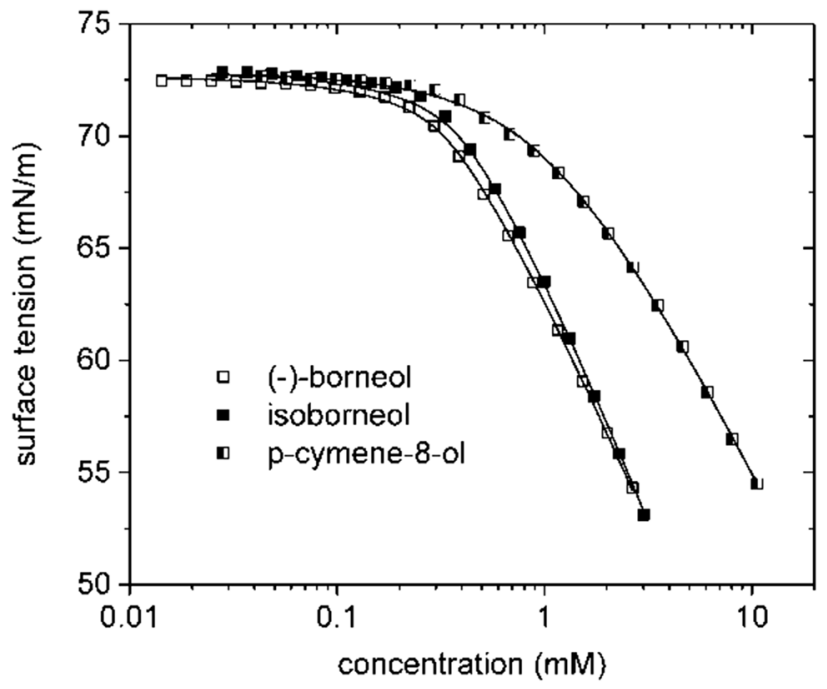

Fig. 5 The values of the surface tension of aqueous solutions of borneol, isoborneol and $p$-cymene-8-ol vs. concentration at $\mathrm{T}=293 \mathrm{~K}$ (points-measured, lines—best fits to the van der Waals adsorption model)

the water-air interface (A) were calculated as a reciprocal of $\Gamma_{m}$ and are also given in Table 1. It follows from Table 1 that the transfer of the (-)-terpinen-4-ol molecules to the monolayer is the least favourable of the seven alcohols for which it was possible to calculate the values of $\Delta G^{o}$. Thus, to compare the adsorption properties of all studied alcohols, in our opinion the best way was to determine the adsorption free energy, $E_{a}$, from the relation (Ivanov et al. 2006):

$K=\alpha \delta_{S} \exp \left(\frac{-E_{a}}{k T}\right)$ 
Table 1 The values of $p C_{20}, \Delta G^{o}$, maximal Gibbs surface excess concentration of MAs $\left(\Gamma_{m}\right)$ and those of the excluded area $(A)$ occupied by MAs at the water-air interface at $\mathrm{T}=293 \mathrm{~K}$

\begin{tabular}{lllll}
\hline Alcohol & $p C_{20}$ & $\Delta G^{o}(\mathrm{~kJ} / \mathrm{mol})$ & $\begin{array}{l}\Gamma_{m}\left(\times 10^{6}\right. \\
\left.\mathrm{mol} / \mathrm{m}^{2}\right)\end{array}$ & $A\left(\AA^{2}\right)$ \\
\hline (-)- $\beta$-Citronellol & 3.280 & -28.16 & 4.58 & 36.3 \\
Geraniol & 3.004 & -26.62 & 4.37 & 38.0 \\
Tetrahydrolinalool & 3.202 & -27.73 & 4.30 & 38.6 \\
Linalool & 2.664 & -24.71 & 3.50 & 47.4 \\
(-)-Menthol & 2.904 & -26.06 & 3.20 & 51.9 \\
(-)-Terpinen-4-ol & 2.392 & -23.19 & 2.25 & 73.8 \\
$\alpha-$ Terpineol & 2.417 & -23.33 & 3.48 & 47.7 \\
p-Cymene-8-ol & - & - & 2.75 & 60.4 \\
Isoborneol & - & - & 3.47 & 47.9 \\
(-)-Borneol & - & - & 3.14 & 52.9 \\
\hline
\end{tabular}

where $\delta_{S}$ is the thickness of the adsorbed layer, $K$ and $\alpha$ are the two adsorption parameters, adsorption constant and minimal excluded area per molecule at the interface, respectively. The mentioned parameters are the function of substrate structure and the medium properties. According to Slavchov and Ivanov (Slavchov and Ivanov 2017), $K$ is the most important parameter related to the change of Gibbs energy of the molecule upon its transfer from the bulk phase to the interface and $\alpha$ is related to the lateral interactions between the molecules in the monolayer. To calculate the adsorption energy from Eq. (3) first the values of $K$ and $\alpha$ were determined by fitting the experimental data of surface tension to various adsorption models. The following models were applied:

a) The Langmuir adsorption isotherm (Langmuir 1918; Hill 1962):

$K C=\frac{\alpha \Gamma}{1-\alpha \Gamma}$

$\gamma_{L V}=\gamma_{0}+\frac{k T}{\alpha} \ln (1-\alpha \Gamma)$

b) The Frumkin adsorption isotherm (Frumkin 1925):

$$
\begin{aligned}
& K C=\frac{\alpha \Gamma}{1-\alpha \Gamma} \exp \left(-\frac{2 \beta \Gamma}{k T}\right) \\
& \gamma_{L V}=\gamma_{0}+\frac{k T}{\alpha} \ln (1-\alpha \Gamma)+\beta \Gamma^{2}
\end{aligned}
$$

c) The Volmer adsorption isotherm (Stanimirova et al. 2011):
$K C=\frac{\alpha \Gamma}{1-\alpha \Gamma} \exp \left(\frac{\alpha \Gamma}{1-\alpha \Gamma}\right)$

$\gamma_{L V}=\gamma_{0}-k T \frac{\Gamma}{1-\alpha \Gamma}$

d) The van der Waals adsorption isotherm (Hill 1962; Jaycock 1981):

$K C=\frac{\alpha \Gamma}{1-\alpha \Gamma} \exp \left(\frac{\alpha \Gamma}{1-\alpha \Gamma}-\frac{2 \beta \Gamma}{k T}\right)$

$\gamma_{L V}=\gamma_{0}-k T \frac{\Gamma}{1-\alpha \Gamma}+\beta \Gamma^{2}$

e) The Helfand, Frisch and Lebowitz (HFL) adsorption isotherm (Helfand et al. 1961):

$K C=\frac{\alpha \Gamma}{1-\alpha \Gamma} \exp \left(\frac{\alpha \Gamma(3-2 \alpha \Gamma)}{(1-\alpha \Gamma)^{2}}\right)$

$\gamma_{L V}=\gamma_{0}-k T \frac{\Gamma}{(1-\alpha \Gamma)^{2}}$

f) The Smith, Ivanov, Ananthapadmanabhan and Lips (SIAL) adsorption isotherm (Smith 1967; Ivanov et al. 2006):

$K C=\frac{\alpha \Gamma}{1-\alpha \Gamma} \exp \left[\frac{\alpha \Gamma(3-2 \alpha \Gamma)}{(1-\alpha \Gamma)^{2}}-\frac{2 \beta \Gamma}{k T}\right]$

$\gamma_{L V}=\gamma_{0}-k T \frac{\Gamma}{(1-\alpha \Gamma)^{2}}+\beta \Gamma^{2}$

In the above equations $k$ is the Boltzman constant, $\gamma_{0}$ - the surface tension of water $(72.8 \mathrm{mN} / \mathrm{m}$ at $\mathrm{T}=293 \mathrm{~K})$ and $\beta$ - the attraction constant known as the interaction parameter. At this point, it should be emphasized that it is difficult to evaluate the suitability of a particular adsorption model for the description of experimental tensiometric data solely on the basis of the quality of fit. The comparison between various adsorption models usually consists of a physical interpretation of the values of adsorption parameters obtained from the best fits of model equations to experimental data. The differences between the values of adsorption parameters obtained for various adsorption models stem from different assumptions made in their derivation. The most popular isotherm is that of Langmuir which, similar to the Frumkin one, corresponds to the localized adsorption, that is to the 
two-dimensional lattice statistics in the Bragg-Williams approximation (Hill 1962). On the other hand, the van der Waals adsorption model, termed also the Hill-de Boer model is derived assuming nonlocalized adsorption of the two surfactant species (de Boer 1953). Nonlocalized adsorption is also described by the Volmer isotherm which is rigorously valid only for the fluid of hard rods adsorbed on a line. The HFL model deserves also attention since it is the equation of state for freely moving hard discs at the fluid interface (delocalized adsorption layer of hard discs in the absence of attraction) (Slavchov and Ivanov 2017). On the other hand, Smith (1967) corrected empirically the HFL equation of state with the addition of an attractive term and obtained the equation of state which was the basis for the derivation of the SIAL adsorption model (Ivanov et al. 2006).

Taking Eqs. (4)-(15) into account, the values of adsorption parameters were calculated and are presented in Table 2. Among employed adsorption models, the Frumkin and the van der Waals adsorption models provide the values of $\alpha$ which are most similar to the values of the minimal area per molecule calculated on the basis of the maximal value of the Gibbs surface excess concentration obtained from Eq. (2) (cf. Table 1). This suggest that the lateral interactions between the adsorbed molecules has to be taken into account for a reliable description of the adsorption of monoterpene alcohols at the water-air interface. Interestingly, the higher is the value of the interaction parameter $\beta$ for the van der Waals adsorption model, the higher is the consistency of $\alpha$ values between Frumkin and van der Waals models, despite the fundamental differences in their derivation. The van der Waals adsorption isotherms calculated for studied MAs on the basis of adsorption parameters given in Table 2. are presented in Fig. 6. Kralchevsky et al. (2003) suggested that the van der Waals adsorption model is the most appropriate for the studies of adsorption parameters of alkanols. Therefore, further calculations of $E_{a}$ through Eq. (3) and based on the adsorption parameters obtained from Eqs. (10) to (11) was carried out. In these calculations the values of the thickness of the adsorbed monolayer were determined for each MA on the basis of: (1) estimated values of van der Waals volume of the MA molecule calculated from the atomic and bond contributions (Zhao et al. 2003) and (2) the values of $\alpha$ obtained by fitting the experimental data to the van der Waals adsorption model. The values of $\delta_{S}$ calculated in this way for all studied MAs are presented in Table 3 together with the values of $E_{a}$ obtained from Eq. (3). As follows from this Table, (-)- $\beta$-citronellol and (-)-terpinen-4-ol show the highest tendency to adsorb at the water-air interface. In the case of (-)- $\beta$-citronellol this is in accordance with the value of $\Delta G^{o}$ and should result from the perpendicular orientation of the molecules of this alcohol at the water-air interface (Fig. 7). In the

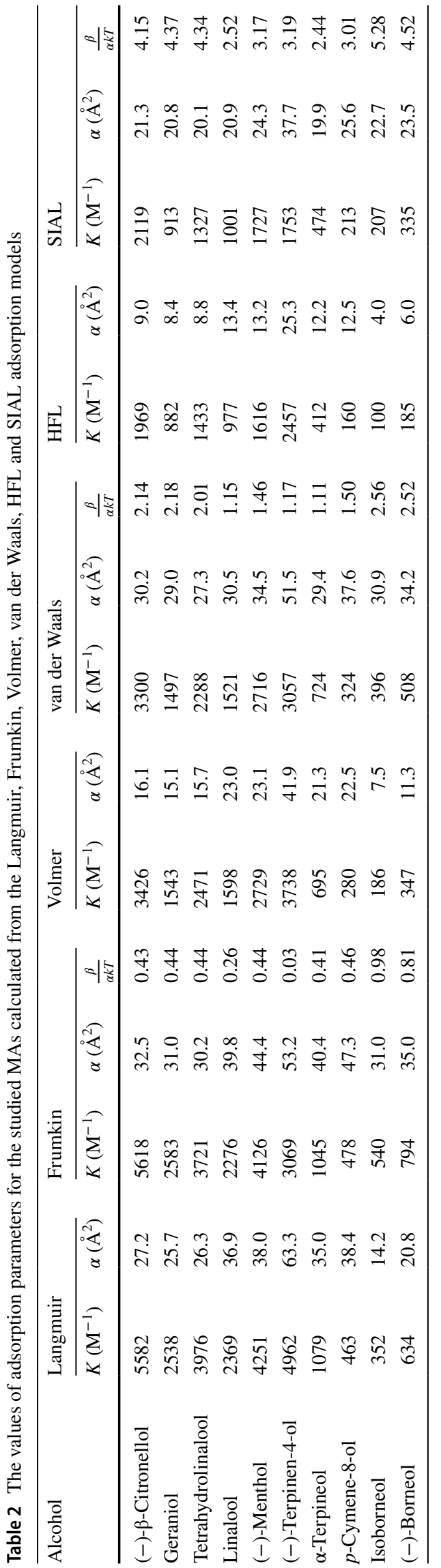




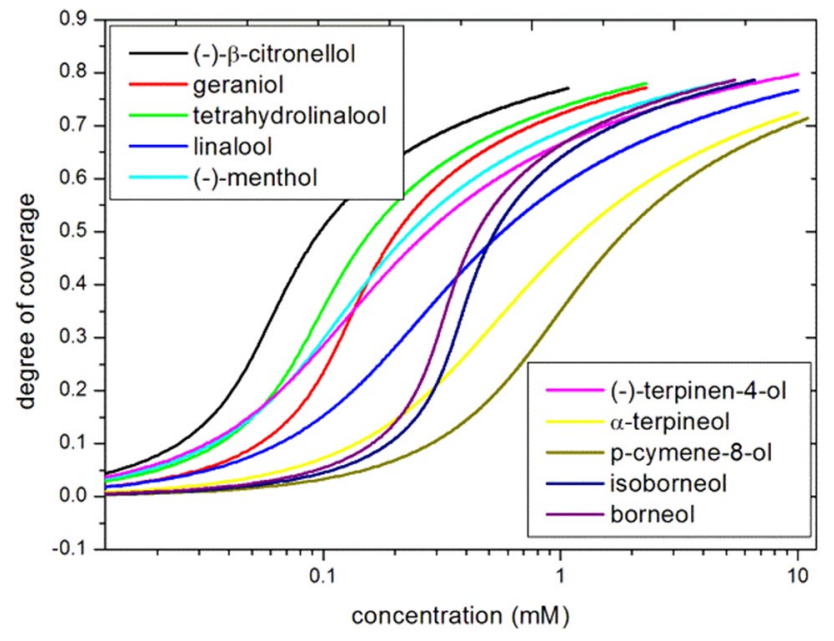

Fig. 6 The values of the degree of coverage calculated on the basis of the van der Waals model for the studied MAs vs. concentration at $\mathrm{T}=293 \mathrm{~K}$

Table 3 The computed values and the literature data of $\log K_{O W}$ of the studied MAs as well as the calculated values of $\delta_{S}$ and $E_{a}$

\begin{tabular}{|c|c|c|c|c|}
\hline \multirow[t]{2}{*}{ Alcohol } & \multicolumn{2}{|l|}{$\log K_{O W}$} & \multirow[t]{2}{*}{$\delta_{S}(\AA)$} & \multirow[t]{2}{*}{$E_{a}(\mathrm{~kJ} / \mathrm{mol})$} \\
\hline & Literature $^{\mathrm{a}}$ & Computed $^{\mathrm{b}}$ & & \\
\hline (-)- $\beta$-Citronellol & 3.91 & 3.38 & 6.02 & -25.14 \\
\hline Geraniol & 3.56 & 3.28 & 6.18 & -23.25 \\
\hline Tetrahydrolinalool & - & 3.51 & 6.76 & -24.21 \\
\hline Linalool & 3.50 & 3.28 & 5.87 & -23.29 \\
\hline (-)-Menthol & 3.40 & 3.20 & 4.99 & -24.80 \\
\hline (-)-Terpinen-4-ol & 3.26 & 2.99 & 3.29 & -25.12 \\
\hline$\alpha$-Terpineol & 3.28 & 2.79 & 5.76 & -21.61 \\
\hline p-Cymene-8-ol & - & 2.19 & 4.08 & -19.90 \\
\hline Isoborneol & 3.24 & 2.71 & 5.17 & -20.29 \\
\hline (-)-Borneol & 3.01 & 2.71 & 4.75 & -20.67 \\
\hline
\end{tabular}

${ }^{\text {a }}$ Determined by RP-HPLC (Griffin et al. 1999)

${ }^{\mathrm{b}}$ Calculated with the ACD/Chemsketch 2012 software (ACD Labs, Canada) case of (-)-terpinen-4-ol, taking into account its structure (Fig. 1) and the value of $\Delta G^{o}$ at the studied interface its molecules should take rather a flat position (Fig. 7). It should be noted that the calculated values of the free energy change of adsorption are quite similar for all of the investigated alcohols and fall in the range between -20 and $-25 \mathrm{~kJ} / \mathrm{mol}$ which is in accordance to the similar size and molecular volumes of these solutes. Kralchevsky et al. (2003) described the adsorption of a homologous series of $\mathrm{n}$-alkanols in terms of the van der Waals adsorption model and found that the free energy change of adsorption for 1 -decanol is equal to $-25.39 \mathrm{~kJ} / \mathrm{mol}$. The respective values found in this study for acyclic monoterpene alcohols (linalool, tetrahydrolinalool, (-)- $\beta$-citronellol and geraniol) are slightly lower than the above mentioned value for 1-decanol and indicate that the addition of a double bond and shifting the hydroxy group from the terminal position deeper into the hydrocarbon chain leads to the decrease of the free energy of adsorption. Similarly, cyclization of the alkyl chain also leads to the decrease of the free energy change of adsorption. The lowest values of this parameter were found for $p$-cymene-8-ol and bicyclic alcohols, which is in accordance to the higher water solubility of the benzene ring and further decrease of the molecular volume due to cyclization, respectively. In general, the absolute values of $E_{a}$ were found to decrease with the decrease of the molecular volume of a solute. This is justified, since the free energy change of adsorption at the water-air interface is associated with the transfer of the molecule from the bulk aqueous phase into the surface region. Thus, it should be related to the relative lipophilicity of a solute. From this point of view it was interesting to compare the obtained adsorption parameters with the literature (Griffin et al. 1999) or calculated values of the octanol-water partition coefficient $\left(\log K_{O W}\right)$. As results from Fig. 8 the tendency of the MAs molecule to adsorb at the water-air interface measured by its adsorption constant $K$, generally increases with the increasing lipophilicity of the MA molecule. However, it is not possible to predict the value of
Fig. 7 Orientation of (-)- $\beta$-citronellol (left) and (-)-terpinen-4-ol (right) molecules at the water-air interface

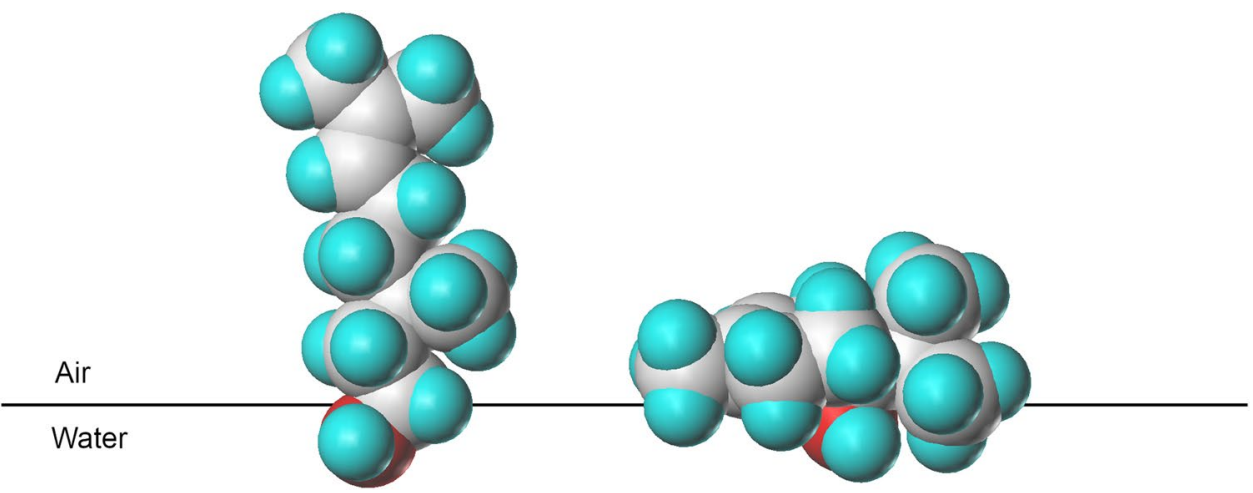




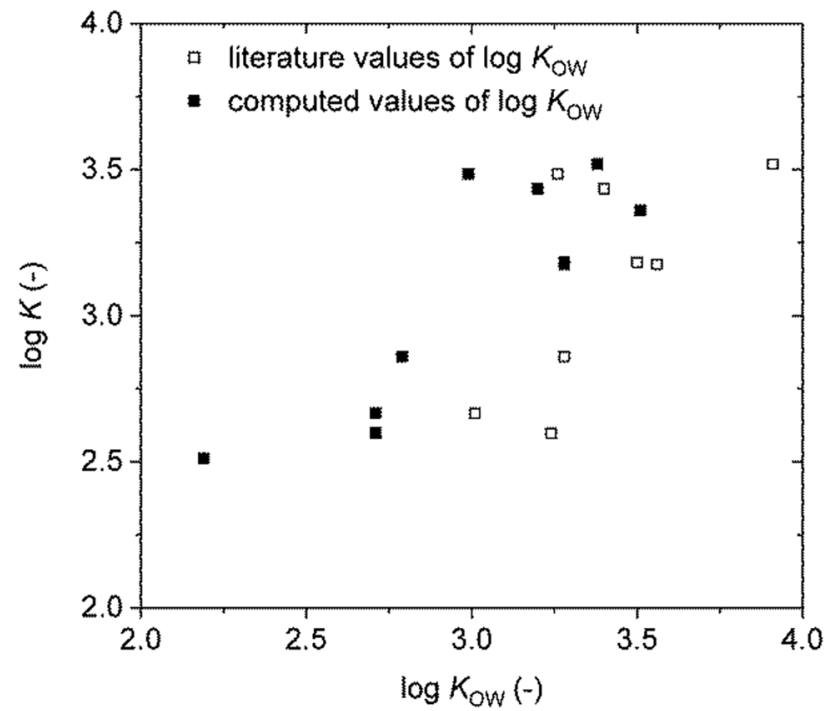

Fig. 8 The values of $\log K$ of the studied MAs calculated on the basis of the van der Waals model vs. the computed and the literature data of the octanol-water partition coefficient, $\log K_{O W}$

the adsorption constant solely on the basis of the $\log K_{O W}$ value of monoterpene alcohol-the values of the adsorption constant of two alcohols with similar values of the octanol-water partition coefficient can differ from each other by up to an order of magnitude (cf. isoborneol and (-)-terpinen-4-ol in Table 2).

Comparison of the values of parameters of the van der Waals adsorption model for the studied MAs with the literature values for 1-decanol (Kralchevsky et al. 2003) leads also to the conclusion that the branching and cyclization of the alkyl chain in the monoterpene alcohol molecule increases the excluded area available per molecule at the interface $(\alpha)$ and decreases the value of interaction parameter $(\beta)$. It was found that monoterpene alcohols with the hydroxy group attached to the acyclic part of the molecule have similar values of $\alpha$, in the range between 27.3 and $30.5 \AA^{2}$. Higher value of $\alpha$ in this group of MAs was found only for $p$-cymene-8-ol. This is probably related to the tilt of the molecule relative to the perpendicular orientation at the interface, caused by the rigidity of $p$-tolyl substituent. The much higher value of $\alpha$ found for (-)-terpinen-4-ol when compared to (-)-menthol can be explained by the relative position of the hydroxy group attached to the cyclohexane ring. The hydroxy group in (-)-menthol molecule is in the equatorial position and allows the plane of a cyclohexane ring to be oriented perpendicularly to the interface. On the other hand, the hydroxy group in the (-)-terpinen-4-ol molecule is in the axial position and forces the plane of a cyclohexane ring to be oriented in parallel to the interface, as shown in Fig. 7.

\section{Conclusions}

In the paper the adsorption constant, the excluded area per molecule at the water-air interface and the interaction parameter were determined by fitting the experimental data of the surface tension of aqueous solutions of the studied 10 monoterpene alcohols (MAs) at $\mathrm{T}=293 \mathrm{~K}$ to different adsorption models. Next the adsorption free energy of studied MAs was determined and discussed in terms of the preferred orientation of the studied MAs at the water-air interface. From the presented data and calculations, it is evident that the studied MAs are effective surface active agents and their tendency to adsorb at the studied interface generally increases with the increasing lipophilicity of the molecule. Also the changes of the minimal excluded area per molecule at the interface, $\alpha$, obtained from the van der Waals adsorption model with the molecular structure of MAs are in quite good agreement with those in the cross sectional area occupied by the molecules perpendicularly oriented at the water-air interface. We have shown that the differences in a molecular structure of alcohols with a similar general formula $\left(\mathrm{C}_{10} \mathrm{H}_{\mathrm{n}} \mathrm{O}, \mathrm{n}=14-22\right)$ may have a substantial impact on their adsorption at the water-air interface. The results of this study may serve as the basis for the investigation of the effect of molecular structure of monoterpene alcohols on their adsorption from systems which are more complex, but also more technologically relevant, such as aqueous solutions of ionic surfactants.

Author contributions AL performed the measurements, made the theoretical calculations and wrote some part of the paper. KS wrote some part of the main text, made figures and tables and corrections of all paper.

\section{Compliance with ethical standards}

Conflict of interest The authors declare that they have no conflict of interest.

Open Access This article is distributed under the terms of the Creative Commons Attribution 4.0 International License (http://creativeco mmons.org/licenses/by/4.0/), which permits unrestricted use, distribution, and reproduction in any medium, provided you give appropriate credit to the original author(s) and the source, provide a link to the Creative Commons license, and indicate if changes were made.

\section{References}

Bicchi, C., Chaintreau, A., Joulain, D.: Identification of flavour and fragrance constituents. Flav. Fragr. J. 33(3), 201-202 (2018)

Bonikowski, R., Paoli, M., Szymczak, K., Krajewska, A., WajsBonikowska, A., Tomi, F., Kalemba, D.: Chromatographic and spectral characteristic of some esters of a common monoterpene alcohols. Flav. Fragr. J. 31(4), 290-292 (2016) 
Bradbury, R., Penfold, J., Thomas, R.K., Tucker, I.M., Petkov, J.T., Jones, C.: Manipulating perfume delivery to the interface using polymer-surfactant interactions. J. Colloid Interface Sci. 466, 220-226 (2016)

Braga, A., Guerreiro, C., Belo, I.: Generation of flavors and fragrances through biotransformation and de novo synthesis. Food Biop. Technol. 11(12), 2217-2228 (2018)

Costa, P., Teixeira, M.A., Lièvre, Y., Loureiro, J.M., Rodrigues, A.E.: Modeling fragrance components release from a simplified matrix used in toiletries and household products. Ind. Eng. Chem. Res. 54(46), 11720-11731 (2015)

D’Auria, M., Lorenz, R., Racioppi, R., Romano, V.A.: Fragrance components of Platanthera bifolia subsp. osca. Nat. Prod. Res. 31(14), 1612-1619 (2017)

de Boer, J.H.: The dynamical character of adsorption. Clarendon Press, Oxford (1953)

Fieber, W., Frank, S., Herrero, C.: Competition between surfactants and apolar fragrances in micelle cores. Colloids Surf. A 539, 310-318 (2018)

Frumkin, A.: Surface tension curves of the higher fatty acids and the equation of condition of the surface layer. Z. Phys. Chem. 116, 466-484 (1925)

Griffin, S., Wyllie, S.G., Markham, J.: Determination of octanol-water partition coefficient for terpenoids using reversed-phase high-performance liquid chromatography. J. Chromatogr. A 864, 221-228 (1999)

He, L., Hu, J., Deng, W.: Preparation and application of flavor and fragrance capsules. Polym. Chem. 9(40), 4926-4946 (2018)

Helfand, E., Frisch, H.L., Lebowitz, J.L.: Theory of the two- and onedimensional rigid-sphere fluids. J. Chem. Phys. 34, 1037-1042 (1961)

Hill, T.L.. An introduction to statistical thermodynamics. AddisonWesley, Boston (1962)

Ivanov, I.B., Ananthapadmanabhan, K.P., Lips, A.: Adsorption and structure of the adsorbed layer of ionic surfactants. Adv. Coll. Inter. Sci. 123-126, 189-212 (2006)

Jaycock, M.J., Parfitt, G.D.: Chemistry of Interfaces. Wiley, New York (1981)
Kaur, R., Kukkar, D., Bhardwaj, S.K., Kim, K.-H., Deep, A.: Potential use of polymers and their complexes as media for storage and delivery of fragrances. J. Control. Rel. 285, 81-95 (2018)

Kralchevsky, P.A., Danov, K.D., Kolev, V.L., Broze, G., Mehreteab, A.: Effect of nonionic admixtures on the adsorption of ionic surfactants at fluid interfaces. 1. Sodium dodecyl sulfate and dodecanol. Langmuir 19, 5004-5018 (2003)

Langmuir, I.: The adsorption of gases on plane surfaces of glass, mica and platinum. J. Am. Chem. Soc. 40, 1361-1403 (1918)

Penfold, J., Thomas, R.K., Bradbury, R., Tucker, I., Petkov, J.T., Jones, C.W., Webster J.R.P.: Probing the surface of aqueous surfactantperfume mixed solutions during perfume evaporation. Colloids Surf. A 520, 178-183 (2017)

Priebe, X., Daugulis, A.J.: Thermodynamic affinity-based considerations for the rational selection of biphasic systems for microbial flavor and fragrance production. J. Chem. Technol. Biotechnol. 93(3), 656-666 (2018)

Rosen, J.M.: Surfactants and interfacial phenomena, 3rd edn. Wiley, New York (2004)

Saeki, Y.: Effect of the fragrance on concentration. Adv. Intel. Sys. Comp. 819, 646-649 (2019)

Sato, Y., Han, J., Fukuda, H., Mikami, S.: Enhancing monoterpene alcohols in sweet potato shochu using the diglycoside-specific $\beta$-primeverosidase. J. Bios. Bioengin. 125(2), 218-223 (2018)

Sell, C. (ed.): The chemistry of fragrances: from perfumer to consumer, 2nd edn. RSC, Cambridge (2006)

Slavchov, R.I., Ivanov, I.B.: Adsorption parameters and phase behaviour of non-ionic surfactants at liquid interfaces. Soft Matter 13, 8829-8848 (2017)

Smith, T.: Monolayers on water: I. A theoretical equation for the liquid expanded state. J. Colloid Interface Sci. 23, 27-35 (1967)

Stanimirova, R., Marinova, K., Tcholakova, S., Denkov, N.D., Stoyanov, S., Pelan, E.: Surface rheology of saponin adsorption layers. Langmuir 27, 12486-12498 (2011)

Zhao, Y.H., Abraham, M.H., Zissimos, A.M.: Fast calculation of van der Waals volume as a sum of atomic and bond contributions and its application to drug compounds. J. Org. Chem. 68(19), 7368-7373 (2003) 